\title{
Coulisses
}

Revue de théâtre

25 | Hiver 2002

Varia

\section{L'écriture de Philippe Minyana}

Transcription de «L'Échange » entre Philippe Minyana et le public

Jacky Castang

\section{CpenEdition}

Journals

Édition électronique

URL : http://journals.openedition.org/coulisses/5987

DOI : $10.4000 /$ coulisses.5987

ISSN : 2546-9460

Éditeur

Presses universitaires de Franche-Comté

\section{Édition imprimée}

Date de publication : 1 janvier 2002

Pagination : 37-55

ISBN : 2-84627-052-X

ISSN : $1150-594 \mathrm{X}$

Référence électronique

Jacky Castang, «L'écriture de Philippe Minyana », Coulisses [En ligne], 25 | Hiver 2002, mis en ligne le 24 octobre 2019, consulté le 15 novembre 2019. URL : http://journals.openedition.org/coulisses/5987 ; DOI : 10.4000/coulisses.5987

Ce document a été généré automatiquement le 15 novembre 2019.

Coulisses 


\section{L'écriture de Philippe Minyana}

Transcription ${ }^{1}$ de « L'Échange » entre Philippe Minyana et le public

\section{Jacky Castang}

\section{Philippe Minyana après la représentation de Pièces le 22 novembre 2001 à Vesoul, au ThéV'. \\ «L'Échange » est une rencontre après la représentation de Pièces avec le public qui le souhaite...}

JACKY CASTANg : "Existe-t-il pour toi des périodes, des phases dans ton écriture, dans ton style? Qu'est-ce qui te nourrit dans une phase d'écriture ? Est-ce qu'il y a des thèmes forts que tu souhaites développer ou est-ce qu'il n'y a pas de période particulière? »

\section{L'inspiration}

Philippe Minyana : «Il y a des auteurs, des artistes qui travaillent toute leur vie sur la même matrice, qui exploitent le même champ, je dirais. Et c'est vrai que je suis quelqu'un qui fait exactement l'inverse, mais ce n'est pas vraiment un choix. Ce qui me fascine dans l'écriture c'est que c'est plus fort que nous, c'est-à-dire souvent le stylo va plus vite que la pensée ; des choses s'installent, s'instaurent, se décident; je dirais ce que l'on a appelé longtemps l'inspiration. Peut-être, ça se décide hors de nous-même, peut-être lorsque l'on est dans une alchimie particulière au moment où la biographie rencontre le monde, où l'âge rencontre la mémoire, je ne sais pas.

Alors c'est vrai qu'il y a différents moments de l'écriture, il y a des pièces qui sont des règlements de comptes ou des choses thérapeutiques: les premières pièces que je n'aime pas beaucoup ne sont pas publiées; et je ne veux pas qu'elles soient publiées, c'est les premiers jets, comme ça.

Et après je suis arrivé à Paris, et c'est vrai qu'on est une famille où on ne lisait pas le journal ou pratiquement pas. Vaguement L'Est Républicain. On habitait la région de Montbéliard, et en arrivant à Paris j'ai commencé à lire le journal tout simplement, lire Libération; c'est pas très original. En 1980 j'ai été frappé par la façon dont ce journal traitait le fait divers. Déjà il y avait une fiction, il y avait l'intérieur du fait divers, du style direct qui donnait la parole à l'assassin ou au témoin, etc. : j'ai été très frappé par cette langue orale que j'ai eu envie de reconstituer. Et ça a donné lieu à ces 
monologues des années 80. J'ai été très influencé par des auteurs allemands, notamment un qui n'est plus très connu maintenant qui s'appelle Achternbuch, et évidemment Peter Hanke et Botho Strauss, que les jeunes allemands d'ailleurs ne connaissent plus. Ils étaient pour moi vraiment des maitres de l'écriture. Plus un auteur français qui s'appelle Michel Vinaver, que j'ai traversé moi en tant qu'acteur, qui ne ponctue pas et qui reconstitue cette parlotte des gens ordinaires et ça j'ai vraiment voulu en rendre compte, voilà. Et alors il y a eu tous ces monologues, peutêtre que certains connaissent ces textes parce qu'ils étaient au programme du bac théâtre l'année dernière et l'année d'avant.

Et d'ailleurs cette année, à signaler qu'il n'y a plus d'auteur vivant au programme du bac. Ça c'est assez violent. Au lieu de supprimer Ibsen, ils ont supprimé Vinaver qui devait me succéder. Enfin bon, c'est une petite remarque en passant, qui signale bien notre société. Et puis après c'est vrai qu'il y a eu une fracture, je dirais dans les années $90, j$ 'ai peut-être digéré la mort de ma mère, le suicide de ma mère, pardon ne pleurez pas, c'est la vie en 80 et j'ai voulu parler peut-être aussi à l'instar d'auteurs comme Thomas Bernhard, comme Beckett qui avaient écrit des formes brèves, denses, rapides, - dramaticules, dramuscules -, et j'ai écrit Les drames brefs, et je vous lirai ce soir Les drames brefs $2^{2}$. Et on s'est fait jeter quand on les a montrés avec Cantarella, parce que j'étais passé de formes logorrhéiques, Chambres ${ }^{3}$ - Inventaires ${ }^{4}$ Les guerriers ${ }^{5}$ - à une parole restreinte. Je passais du maximalisme au minimalisme, de la logorrhée au laconique. Je changeais radicalement de style et c'est toujours compliqué quand on change de statut parce que les gens se disaient : "Tiens le pauvre Minyana il ne sait plus écrire." C'était clairement dit et je préfère personnellement ces drames brefs-là à ce qui a suivi, à ce qui précède. Donc j'ai travaillé sur les pièces de deuil, sur la situation de l'homme dans le monde je dirais. De façon pas réaliste, pas naturaliste qui sont des genres que je n'aime pas trop : plutôt la stylisation, la métaphore, l'allégorie plus que le personnage, la figure plus que le personnage et donc on pourrait appeler ça par coquetterie, mes fantômes, enfin des espèces d'humanoïdes, d'êtres qui apparaissent pour disparaître.

\section{Le fragment, la langue, la fiction}

C'est pas des pièces forcément linéaires, c'est pas des pièces forcément dans la structure classique évidemment. Ça travaille par fragments, je crois que le fragment, sans vouloir être professoral, ni pédant, je crois que le fragment désigne fortement la littérature d'aujourd'hui. Je dirais qu'il y a des auteurs qui me passionnent, comme Claude Simon, je ne sais pas si vous connaissez, il y a un de ses bouquins qui vient de sortir Le tramway au printemps, je crois. Et Claude Simon est un monsieur qui a presque 80 ans, qui a eu le prix Nobel en 1986 qui parle de la guerre et de sa biographie. Il «tricote » les deux. Je suis très très passionné par des œuvres comme ça : la langue, je dirais que la langue entraîne la fiction et pas l'inverse.

Ce qui me fait chier vraiment c'est quand mon éditeur me dit, il faut résumer les pièces et marquer combien d'hommes, combien de femmes, parce que parait-il les gens veulent ça. Mais je trouve ça monstrueux parce que ça réduit le poème. C'est aberrant. Je veux dire, ils n'ont qu'à se démerder les amateurs s'ils veulent jouer. Un homme peut jouer une femme et une femme peut jouer un homme. Il faudrait peutêtre savoir que le théâtre c'est la magie, l'illusion et qu'on fait pas du sitcom quoi. Donc après il y a eu La Maison des morts ${ }^{6}$, Pièces $^{7}$, qui sont des pièces, des fables sur le 
monde je dirais, où tout d'ailleurs, tout repose sur des histoires vraies. Je suis toujours fasciné par les faits biographiques, mais surtout par des histoires qui arrivent de l'extérieur.

\section{Pièces, un mystère laïc}

1 Et quand Cantarella m'a demandé d'écrire Pièces pour des raisons diverses que je ne vous raconterai pas, c'est trop long, j'ai immédiatement pensé à un fait divers que j'avais lu dans le journal, dans Libération toujours, qui est un journal très discutable par ailleurs, qui est devenu un journal de droite, mais bon ça c'est une autre histoire. Il y a dix ans, est arrivée cette histoire de ce vieil homme qui avait accumulé tant d'affaires dans son appartement dans le vingtième arrondissement, qu'il n'arrivait plus à y entrer. Et puis il y a eu une fuite d'eau et la police y est entrée par effraction, et non seulement la police mais aussi les agents de la propreté qui ont pété les plombs et qui ont tout jeté par la fenêtre dans une benne, non seulement les ordures, mais aussi les choses précieuses, les documents, les collections, et les meubles aussi qui ont été vendus aux enchères aux commerçants du coin qui ont dû rendre ensuite les objets au monsieur. Donc ça a fait l'objet de plusieurs articles dans les journaux. Je me suis souvenu que j'avais gardé ce fait divers. Donc, je me suis précipité dessus, et j'ai fait une espèce de symphonie, j'ai composé avec d'autres histoires que j'avais également prélevées dans les journaux, pour construire une espèce de fresque à partir de cet itinéraire, de ce périple de cet homme et après j'ai repensé à des choses archaïques du théâtre : les mystères laïcs; ou comment on assiste à la chute de l'homme, à la chute de l'ange, grandes mythologies de nos civilisations occidentales. Voilà comment ça travaille, la commotion se fait, la brûlure se fait et comment j'organise ma partition je dirais, parce que c'est vrai que ce sont des partitions. Robert l'a dit hier à certains d'entre vous qui étaient déjà là mardi, beaucoup de didascalies, donc d'indications de scènes qui font comme une sorte de mise en scène qui accompagne le texte, du roman qui accompagne l'histoire dont j'ai besoin pour écrire. Après le metteur en scène en fait ce qu'il veut, il le garde ou il ne le garde pas; Robert en a gardé une bonne partie, les deux tiers, en tout cas. Lorsque je vous lirai ce que je viens d'écrire ces trois jours à l'hôtel à Vesoul, ce ne sont que didascalies avec des "incrustes" de petites répliques. Dans le train il y a eu une première étape, et là, à l'hôtel du Lion il y a une deuxième étape, c'est-à-dire tout à coup les choses s'affinent, se précisent. Je me réveille dans la nuit je me dis "Ah, merde il faudrait que je rajoute ça". J'étais au restaurant tout à l'heure et je vois apparaître les "créatures" de plus en plus nettement, en plus je sais pour quels acteurs c'est écrit. On dit: "on est tout seul quand on écrit." C'est pas vrai parce qu'on prévoit le projet futur, c'est pour la scène, donc il y aura une troupe qui va s'emparer de ce que vous avez pondu et ça, c'est formidable. C'est peut-être la raison pour laquelle je n'écris que du théâtre quasiment ou alors quelques accidents de récits fragmentaires d'ailleurs. Voilà !»

\section{Sens et partition}

JACKY CASTANg : "Quand tu parles de "partition" est-ce que pour toi toutes les phrases que tu écris font sens? Est-ce que tu peux admettre que le sens puisse t'échapper dans un 
rapport à la musique, à la poétique, que tu puisses écrire une phrase où le rapport à la musique est plus important que le sens de la phrase? »

Philippe Minyana : "Non, Non, quand même pas, je ne fais pas ça. Le sens m'importe évidemment, mais je pense aussi que le son fait sens. »

JACKY CASTANg : "Prioritairement? »

Philippe Minyana : "Je ne pourrais pas répondre aussi nettement, je sais pas. Je dirais que la phrase me devance, je l'entends, je la récupère. C'est vrai que je choisis souvent des mots pour leur sonorité, mais ça tous les écrivains le font. Je crois que ce n'est pas très original. En revanche, j'ai envie de raconter une histoire. Je crois que Pièces c'est une histoire, il y a la chute d'un homme comme ça en neuf tableaux avec un chœur d'habitants qui vient commenter l'histoire. Il y a là une structure, je travaille comme un artisan, je fais des plans, constitue des dossiers avec plein d'articles que j'utilise ou pas, d'ailleurs. Mais, il y a un travail préliminaire en amont. Je ne peux pas écrire comme ça. Quand on me propose une commande, il y a toujours un flottement épouvantable de dix jours où j'ai des tas de troubles physiologiques parce que je me dis que je ne sais plus écrire. Est-ce que le miracle va cesser ? Ça y est, c'est fini, j'ai toujours peur. Et, c'est vrai qu'il y a toujours à chaque écriture le même flottement, le même trouble, la même anxiété et j'ai l'impression qu'il faut que je réinvente chaque fois la chose, et au bout d'un moment, après il y a l'écriture comme si c'était une espèce de truc qui était là dans l'espace et d'un seul coup se précipite et prend les choses en main. Je dirais, et j'ai l'impression, comment dire ? que je suis dédoublé que c'est quelque chose qui m'échappe partiellement. C'est très bizarre. »

\section{Le théâtre, une parole à dire}

JACKY CASTANg : "Pour toi, est-ce qu'il existe une parole à dire, est-ce que le théâtre c'est cette recherche là d'une parole qui a cette spécificité d'être plus tard dans la bouche d'un comédien, d'un acteur. »

Philippe Minyana : «Ah ! Oui, oui, oui ça c'est clair que c'est mon souci, je le dis, je le profère, je dis toutes les voix je fais tous les... »

JACKY CASTANg : "T'écris et tu parles. »

Philippe Minyana: «Ah! oui, oui, oui. À l'hôtel d'ailleurs je me demandais si les clients de l'hôtel ne se sont pas demandés si j'étais pas un peu fou. Dans la chambre 24, il y avait un mec qui profère : "voix dans les larmes", Et je disais le texte tout l'après-midi et j'écris à la main et je n'ai pas d'ordinateur, et ça, vraiment, ça commence à devenir pénible, mais je n'arrive pas à me brancher sur les machines. Je n'ai même jamais eu une machine à écrire. Je me fais taper mes textes et quand on me donne mon texte tapé, ah ! c'est un plaisir physique, enfin épidermique, comme ça à le trouver. Alors on me dit que je suis noir et pessimiste, et je dis toujours mais je suis toujours en deçà de Skakespeare, Sénèque, Euripide qui racontent des horreurs absolues beaucoup plus fortes que je ne pourrai jamais en écrire. Et puis, je pense que si on cadre le réel, ce qui est mon propos, si on cadre le réel, on cadre l'humain dans sa vie ordinaire, et la vie ordinaire n'est faite que d'accidents. Moi je suis frappé, peut-être parce que c'est ma faute, parce que j'écris des choses sur l'humain, que les gens viennent se confier à moi. J'arrive au Théâtre Dijon Bourgogne où je suis nommé, où je travaille, il y en a une qui entre et qui me dit : "Oh ! là, là ! Ma grandmère va mourir, c'est affreux", et elle me décrit en détail l'agonie de la grand-mère. 
L'autre qui arrive, qui me dit: "Mon palmier est en train de mourir", l'autre qui dit : "Oh, là, là ! Ma meilleure copine qui s'est suicidée". C'est tous les jours que ça arrive ces choses-là. J'entends très fort ça, très, très fort. J'appelle ma sœur qui me dit que son mari ne va pas bien, je raccroche. Mon frère m'appelle et c'est du même acabit. Je veux dire que c'est ça la vie. L'art ne se fait pas sur du rose, je crois, c'est pas possible. Et même, je dirais, les sitcom à la télé pour les mamies à 13h30. Il m'arrive de regarder en ethnologue, mais il n'y a que des tentatives de suicides, des machins d'avocats et de divorces, d'accidents de voitures, de maladies d'enfants, de maladies de femmes; enfin je veux dire, si on retient le réel, on le retient dans son entier voilà. Et alors, ce qui serait immoral et politiquement dégueulasse, ça serait que je fasse de la compassion, de la plainte, du sérieux solennel, et ça non. Je pense que dans mes pièces on peut toujours rire. Je veux qu'on rie. Quand on rit à mes pièces, je suis ravi. Et je fais toujours en sorte qu'il y ait l'alliance du grotesque et du tragique, toujours. Je prends à mon compte la phrase de Victor Hugo dans la préface de Cromwell qui dit qu'il faut toujours le grotesque et le sublime. Je pense que je réponds à ça toujours et c'est une façon de ne pas me complaire non plus dans des histoires qui sont inévitablement violentes. »

JACKY CASTANg : "Sur cette première partie, puisqu'on va aborder Pièces maintenant d'une manière plus précise en référence à la représentation, vous avez une question pour Philippe?»

\section{Pièces, la mise en scène}

PUBLIC : "Disons qu'à propos du rire, c'est pas un rire franc quand même, que vous avez produit, que Pièces a produit. II y a plutôt des moments où on est déconcerté, non pas par la mise en scène, c'était très bien tous ces acteurs qui venaient répéter, vous venez d'expliquer pourquoi, mais la tragédie elle est vraiment présente. »

Philippe Minyana: «J'ai pris le fait divers au moment où il est expulsé de son appartement à Paris. Et alors ce qui m'a vraiment frappé à la première à Paris, parce que je n'avais pas vu beaucoup le travail, je n'étais pas là, ce qui m'a beaucoup frappé, c'est quand on casse le mur, qui est une idée de mise en scène magnifique, je trouve, et que Lotta dit ensuite : "Venez sur ces ruines vous réunir". J'ai trouvé que ça nous parlait très fort du monde d'aujourd'hui évidemment. J'ai seulement compris moimême, en tant que spectateur, ce que la pièce avait écrit toute seule. J'avais presque envie de dire : "Ce n'est pas vraiment comme ça mais c'est quelque chose comme ça aussi" »

JACKY CASTANg: " Il y a des idées; on peut parler aussi d'effets de mise en scène, mais c'est peut-être plus péjoratif, qui sont à ton avis un vrai prolongement du spectacle, comme par exemple la vidéo, la bande-son qui reprend du texte, des choses comme ça, ou est-ce que tu peux toi te dire : "non ça, ça nous éloigne du texte." »

\section{Un projet vertueux}

Philippe Minyana : «C'est le boulot de Cantarella qui collabore au mien. Même s'il y a des endroits que j'aime plus ou moins, je suis très content de voir le travail de l'autre qui complète le mien, je trouve ça émouvant. En plus ça fait vingt ans qu'on bosse ensemble. On a fait Inventaires en 87 ; et de 87 à maintenant on en arrive là. C'est surtout à lui que j'en jette des fleurs. C'est que la mode veut, surtout à Paris, vous ne 
vous rendez peut-être pas compte en province, à Paris, si on n'a pas une vedette de la télé dans un spectacle, c'est très difficile de monter le spectacle. Alors le projet de Cantarella je le trouve vertueux : il y a des acteurs qui sont des anciens élèves de Robert, des acteurs de Dijon parce que nous sommes à Dijon dans le théâtre, des amateurs parce que dans ma pièce c'est marqué "des habitants qui parlent". Il l'a pris au pied de la lettre, il a dit : "Ah! des habitants très bien! Ce sera des gens de Paris, des gens de Vesoul, des gens de Besançon. Puis après la tournée, on verra. S'il y a une tournée."

Bon, donc je trouve qu'il y a un truc vertueux là-dedans dans ce travail artisanal et dans le geste politique que fait Cantarella; engagement qu'il a eu tout l'été dans ce Théâtre Ouvert où moi j'ai commencé il y a vingt ans, où ils m'ont fait trois créations cette année. Je veux dire tout ça est un ensemble de gestes artistiques. Moi, souvent, je suis plus touché, dans le travail d'un artiste, par le projet que par le résultat. Et là, le projet me plaît.

Après dans le résultat, je trouve qu'il y a des choses très gonflées, très fortes qui peuvent dérouter le public. Comme la scène qui est écrite essentiellement en didascalies, des indications de scène, quand tout à coup il y a un seul acteur qui dit toute la scène, et elle se joue en décalé. C'est un peu compliqué pour le public, mais je trouve que c'est un geste artistique magnifique et fort. C'est comme un poème, avec la force du poème et en même temps. Alors évidemment on choisit soit d'écouter le narrateur, soit de regarder les acteurs, alors évidemment ça peut troubler le spectateur. Mais en même temps, moi je dis toujours, et il n'y a pas que moi qui le dis, que l'art est fait pour troubler et pas pour consoler comme dit Jean-Marie Piemme, qui est un collègue à moi, un auteur belge. Il dit: "Si vous voulez être consolé vous allez à la pharmacie ou à l'église, mais pas au théâtre." L'art en général ça trouble. On régresse à mort en ce moment, la télévision, le cinéma, les médias enfin toutes ces choses d'écran banalisent tout.

Cet été, à Paris, j'ai suivi le festival Bergman. J'ai vu un film que je n'avais jamais vu, qui s'appelle Le Silence. Et je me suis dit, c'est pas vrai, on a osé en 1960 produire un truc pareil. Il n'y a aucun producteur aujourd'hui qui produirait Le Silence de Bergman, qui est un film incroyablement génial. Ce monde dans lequel on est, je m'y sens très mal, je dois dire, très mal; dans cette société commerçante où tout est banalisé, les gens viennent s'asseoir dans un théâtre et veulent rigoler; et moi je pense que le théâtre est là pour alarmer, pour empêcher de dormir. Non pas que je dis : "Tiens, ils ne vont rien comprendre, bien fait !" Non, pas du tout. J'ai envie qu'on me comprenne bien sûr, je ne fais pas exprès de rater une marche, un truc de sens ou d'explication, c'est pas volontaire. Moi, j'ai envie que les gens me comprennent ; j'ai pas envie de faire un théâtre pour quinze personnes, non, même si c'est celles-là qui vont peut-être plus vite comprendre, mais peu importe. Le service public, et des gens comme Jacky Castang, que je salue au passage, et que je remercie violemment parce qu'il fait un travail magnifique, cet homme, ça sert à ça. Ça veut dire que, si je vous propose mon poème et bien je viens vous voir après et, je vous dis : "Voilà j'ai voulu faire ça, ça, ça" ; et si le metteur en scène il n'est pas là, je peux dire en gros ce qu'il a voulu faire. Et je suis là pour répondre à vos questions voilà. Et je vous ai vus avant, je vous ai vus après, et je trouve que ça, ça négocie avec les gens pour qui j'écris, qui est le public. Voilà. » 


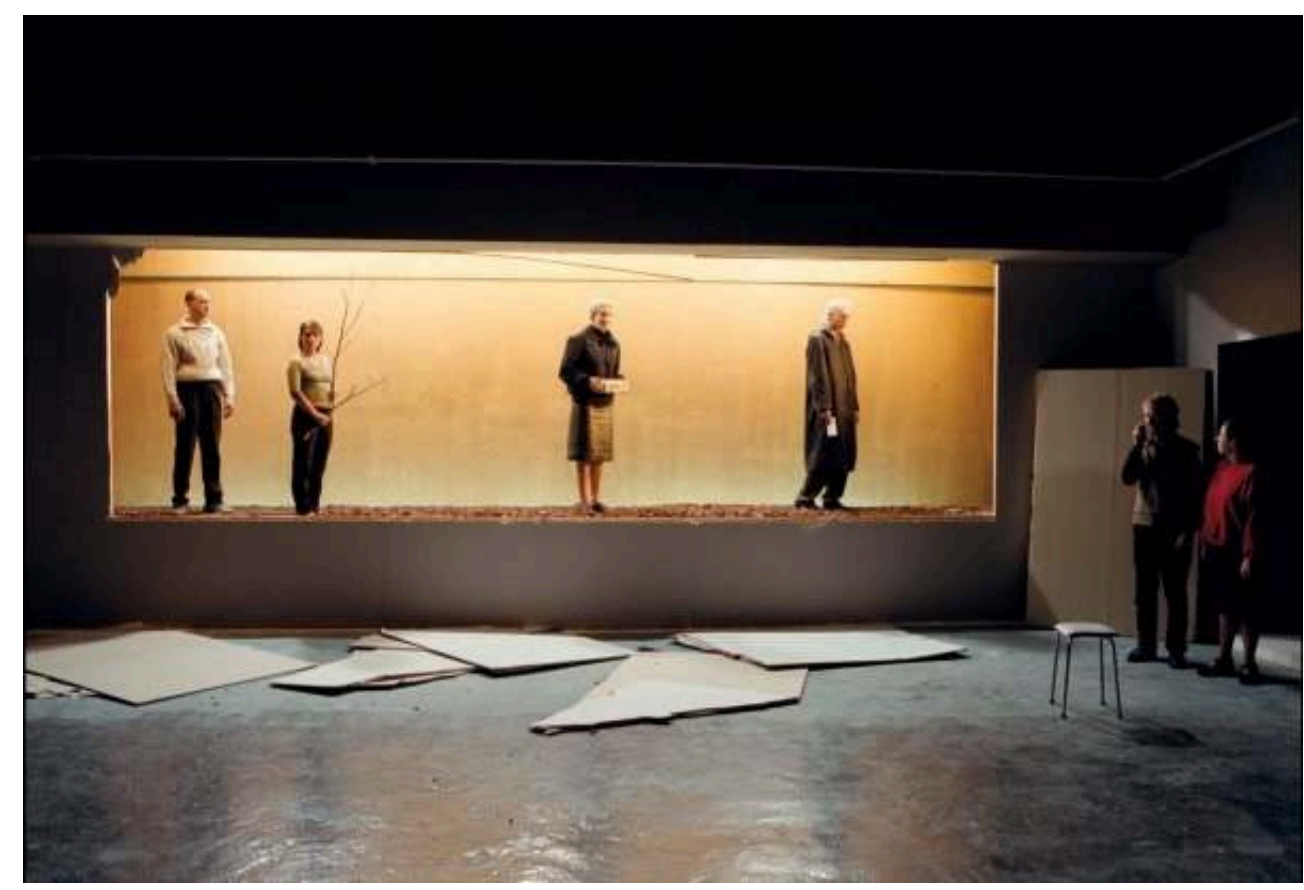

Cliché site web Minyana

PUBLIC : "Vous avez dit Robert Cantarella m'a commandé une pièce pour $X$ et $X$ raisons, est-ce que vous pouvez dire... »

\section{Pièces, une commande de Robert Cantarella8}

Philippe Minyana : "Oui, oui, c'est pas un secret; simplement c'était parce que ça s'est pas fait. Au départ, il voulait monter une pièce de Calderon qui s'appelle $L e$ Prince Constant. C'est une pièce de théâtre baroque, à laquelle j'ai participé pour une nouvelle traduction en français de l'espagnol baroque, je parle pas espagnol, mais la personne qui le parlait, Jean-Jacques Préau était hispanisant. Bon, même si mes ancêtres sont espagnols, d'une certaine partie du côté de mon père, je ne parle pas cette langue, en tout cas pas assez. Et donc, il voulait monter ça, et il m'a dit: "En regard du Prince Constant peux-tu écrire une autre pièce ?" Il a lu Pièces, qui est un gros morceau, parce qu'il a fait plein de coupes. Entre le texte initial et le spectacle il y a pas mal de coupes parce que ça aurait duré 3 h30. Donc, il a fait des coupes, et en regard de la pièce qu'il a reçue, il m'a dit : "Je ne peux pas monter les deux, sinon il y une " prod» énorme. Je ne trouverai jamais l'argent pour monter les deux." Donc il montera plus tard Le Prince Constant qui parlait de la résistance et du sacrifice. Le Prince Constant ça raconte l'histoire ; et ça n'a jamais été créé en Europe, uniquement le cinquième acte par Grotowski. Un prince espagnol a perdu la bataille contre un prince arabe et dit: "Puisque je suis victime, je suis vaincu, et bien je reste sur ma terre (l'enclave espagnole de Cunta au Maroc) et vous me traiterez comme de la merde." Donc, ils lui mettent des épluchures sur la gueule, ils lui pissent dessus, ils lui tordent un peu tout, et il accepte la mort comme Job sur son fumier, voilà la fable. Donc j'avais trouvé comme répondant, aujourd'hui, un homme qui est différent, qui fonctionne autrement, et qui va à sa chute. Donc, c'était une sorte de réponse. Mais plus tard il montera peut-être Le Prince Constant qui est une pièce très codée, baroque, il faut parler de Mars, de Vénus ; les jeunes premiers sont chiants, c'est tous les codes 
du théâtre classique baroque espagnol. Voilà la raison pour laquelle je n'en parlais pas, parce que c'est des anecdotes qui ne sont pas très, très intéressantes puisque ce n'est pas encore réalisé. »

\section{Pièces, un théâtre forain, un théâtre de base}

JACKY CASTANg : "Si on revient précisément sur la représentation de mardi, si on parle du traitement de l'espace, il y a quelque chose qui m'a étonné moi, c'est cette petite maison, qui était du côté jardin. ${ }^{9}$

Philippe Minyana : « La maison des nains »

JACKY CASTANg : "Et qui était là-bas, et qui était dans un espace plutôt signifiant, un espace réaliste. Est-ce que ce n'était pas quelque chose qui était de l'ordre de l'incohérence? C'est quelque chose qui était en présence, en permanence et qui avait plutôt une fonction de troubler. Mais c'était aussi peut-être un objectif recherché. »

Philippe Minyana : «Ah ! ça alors là je... »

JACKY CASTANg : "Tu ne peux pas en parler peut-être... »

Philippe Minyana : " Moi je n'ai pas assisté aux répétitions et il faudrait la dramaturge Marie Pia-Bureau ou le scénographe Philippe Quesne ou Cantarella ; c'est pas moi qui ai décidé qu'il y ait une maison de chez Leroy Merlin à gauche. On peut dire plein de choses comme ça au pif, que c'est l'endroit d'où ils sortent, l'endroit d'où ils arrivent, l'endroit où on range tout, où il $\mathrm{y}$ a les accessoires. Comme c'est une pièce où il $\mathrm{y}$ a huit acteurs seulement et des amateurs, ils jouent ces huit acteurs au moins trente personnages. Donc ils n'arrêtent pas de sortir, de se changer; les acteurs passent presque à vue d'un truc à l'autre. Et donc il y en a un qui met des perruques, les autres qui les enlèvent; ils se mettent un autre vêtement et ils deviennent un charcutier. Moi j'aime bien ce côté forain, j'aime beaucoup, beaucoup cette idée. C'est pour ça que je me permets, quand j'écris pour Cantarella, de mettre une palanquée de personnages parce que je sais qu'il va s'en débrouiller et que ça va devenir, enfin, le théâtre de base, je dirais forain, on joue tout. C'est comme Peter Brook quand il joue L'homme qui. Le mec, il a une couverture sur les genoux, il devient malade ; il l'enlève, il se lève, il prend une blouse de médecin, il la met, il devient médecin. Et tout ça, à vue du spectateur. Moi, j'aime beaucoup ça, qu'on voie la coulisse, qu'on voie les coutures, je trouve ça très bouleversant, parce que c'est l'essence même du théâtre, je dirais. Le simulacre est avoué. J'aime bien cette idée-là. Donc, je ne peux pas trop te répondre, si ce n'est que, je pense que le metteur en scène, comme l'écrivain, a des visions.

\section{Visions du metteur en scène}

Depuis le début, il s'est obstiné à avoir cette petite maison. Le scénographe a résisté, je sais. Moi, on m'en a parlé, j'ai dit : "Ah bon! Pourquoi cette petite maison", et puis ça le titillait il voulait cette petite maison. Il s'est mis cette petite maison; il s'est mis aussi le plan du quartier devant le public. Bon c'est des obsessions de... je sais pas. C'est des visions de metteur en scène aussi, et je les respecte, même si ça ne me passionne pas plus que ça. Je respecte le travail de l'autre, puisque j'écris pour l'autre, donc j'accepte le jeu. Et je veux dire: c'est un spectacle de qualité, un spectacle très intelligent, très fort, très pertinent, très gonflé. Je préfère ça à de la 
daube moyenne quoi. Là, c'est un autre sens, il écrit, il fait son écriture à côté, c'est deux grammaires côte à côte qui fonctionnent plus ou moins bien, toujours et j'aime bien : on est dans le jeu et on est au théâtre. Le théâtre c'est fragile, ça bouge, c'est mobile, c'est un endroit qui n'est pas fixé, figé. Moi, quand je vois les metteurs en scène, des metteurs en scène très célébrissimes qui ont des milliards pour faire des spectacles... Moi quand j'étais acteur, j'ai participé à ce genre de production. On arrive le premier jour, le costumier est là, le maquilleur est là, le scénographe est là et tout est fait. Le décor c'est fini, la musique elle est faite, les costumes ils sont faits et au bout d'un moment, on se fait chier. Quesne et Cantarella ont fabriqué le décor au jour le jour. C'est-à-dire comment faire disparaître la femme qui se..., cette allégorie de l'échec : elle est avalée par la terre puisqu'elle ne sert plus à rien. Comment faire? Donc il fallait trouver des solutions; ça s'est trouvé au jour le jour. En fait, quand je suis arrivé au début, ils avaient un grand nombre de bouquins. Comme je parle souvent des miniatures du Moyen Âge, de la peinture parce que j'aime beaucoup ça, ils avaient plein de bouquins du Quattrocento, des Primitifs. La scène où le mur est cassé, ce qu'on découvre est exactement comme dans une miniature sans profondeur, sans perspective. Les figures sont comme en aplat sur ce mur doré, c'est doré en fait, c'est pas jaune, c'est doré comme les petites miniatures qu'on voit du haut Moyen Âge. Et puis, aussi, quand les amateurs, à la fin, viennent parler disant qu'il a retrouvé ses enfants, etc., ils sont..., enfin c'était moins flagrant à Vesoul, mais plus flagrant à Paris à cause des dispositifs. Il n'y a que des visages éclairés, comme les anges dans les tableaux de la Renaissance. Donc il y a des petites traces, comme ça, culturelles, des petites références, des clins d'œil comme ça qui font qu'on voit le travail, comment ça s'est sédimenté. Comme pour moi, comme pour l'écriture, finalement. »

\section{C'est quoi la modernité ?}

JACKY CASTANg : "Robert Cantarella a insisté aussi beaucoup sur la différence des comédiens, de leur histoire. II parlait aussi, par rapport aux dix amateurs, lorsqu'il les cherchait, d'avoir dix sonorités complètement différentes. Est-ce que, sur ce point, c'était déjà quelque chose qui existait dans ton écriture ? Est-ce que tu penses qu'il n'y a pas eu, mais le mot n'est peut-être pas bien choisi, d'excès, comme par exemple sur le jeu du propriétaire. »

Philippe Minyana: "Je trouve ça très très juste et je lui ai donné exactement la référence, parce qu'on pique plein de trucs quand on travaille. Quand j'écris j'ai Dostoïevski pas loin, Peter Hanke, Botho Strauss, Nathalie Sarraute, puis tous les jeunes Japonais qui écrivent et je n'arrête pas d'ouvrir ces livres. Et Cioran évidemment, mon cher Cioran : "Les Cahiers". "

JACKY CASTANg : " Il y a d'ailleurs des citations de Cioran »

Philippe Minyana: "Oui, oui sans arrêt et de Hanke, et de Dostoïevski. Et donc, j'ouvre, et puis il y a une phrase, et paf, ça fait écrire. Et le propriétaire c'est exactement un personnage de Crimes et châtiments qui poursuit Raskolnikov. D'abord, il lui fait des grâces et ensuite le coince et lui dit: "C'est vous qui avez tué." Il passe, comme ça, de caresses en insultes sans arrêt, il le suit dans la ville etc, etc. Ça m'a beaucoup frappé, ce personnage ambigu et en resserré, bien sûr, parce que ce n'est qu'une scène; j'ai fait un clin d'œil en tout cas à ce personnage dostoïevskien. D'ailleurs, je l'ai dit à Philippe Vieux qui a dû sans doute consulter le bouquin. Donc, ce qu'il y a de beau dans l'écriture, dans l'art en général, c'est comment on travaille 
avec les autres, comment on s'inscrit dans les écritures du vingtième siècle, comment les peintres s'inscrivent dans la peinture du vingtième siècle. Je trouve qu'on ne peut pas écrire pour soi pour se faire plaisir c'est pas une branlette. C'est un truc qui est de l'art donc c'est une brûlure, une commotion, et il faut travailler avec ça, avec ce qui est avant nous, pressentir peut-être ce qu'il y aura après. Moi, je me pose toujours la question, ça va paraître un peu naï, un peu con de dire ça, mais je le dis quand même, je pose tout le temps la question quand je travaille: "C'est quoi la modernité ?" Actuellement, je lis beaucoup de textes, parce que je dirige le comité de lecture du Théâtre de Dijon et je suis très frappé par deux choses : d'une part, les imitations, il y a des faux Sophocle, des faux Marlowe en pagaille en ce moment, beaucoup de pastiches, avec un langage d'avant, ancien, solennel, etc. grandiloquent. Il y a aussi les logorrhées, l'aveu, le journal intime, etc. Il n'y a pas de forme, on ne sait pas qui parle, pourquoi il parle, à qui il parle, où il est, on ne sait rien, et c'est comme ça, ça sort, c'est de la diarrhée verbale. Je ne critique pas, je constate, c'est tout. Donc il y a peu d'écritures qui me paraissent révéler l'aujourd'hui ; et le fragmentaire me paraît juste. Alors, j'ai cité Claude Simon, mais je pourrais citer plein d'autres; et des romanciers aussi qui sont aussi ma nourriture. Je pense à François Bon qui vient de sortir un bouquin récemment chez Verdier, il y a un mois ou deux qui s'appelle Mécanique, qui est un très très beau livre, justement, où il fait la nique à la biographie. Pourquoi ? parce que c'est du fragment, ce n'est pas chronologique. Il ne dit pas : je suis né en Vendée, mon papa était garagiste, etc. Puis on a acheté telle voiture, puis on a eu telle voiture toute notre vie, puis je me suis marié, j'ai fait des gosses, etc. et mon frère aussi. Non, non, pas du tout. Il met les titres, il met par exemple : "maison", "ressemblance", "hangar", "carrefour", "couloir", puis il développe chaque fois. Ce qui fait qu'on a une série de fragments à la suite les uns des autres, ce qui fait qu'on a une composition musicale symphonique. Ce qui nous manque, c'est un courant. On admire les artistes des années 10, 20,30, des années décisives. Quand on voit les architectures, des mecs entre 12 et 20 , on hallucine, ça reste futuriste toujours, la musique aussi, je veux dire toutes les inventions. Quand on pense que Les Demoiselles d'Avignon de Picasso, c'est de 1912. Quand j'ai vu cette merde de film Le Titanic à la télé, l'autre jour, elle amène la toile de Picasso, et son copain, son fiancé dit : "on n'en parlera jamais de ce mec-là, c'est nul, c'est de la daube, c'est mauvais, vous allez pas acheter ça", etc. » 


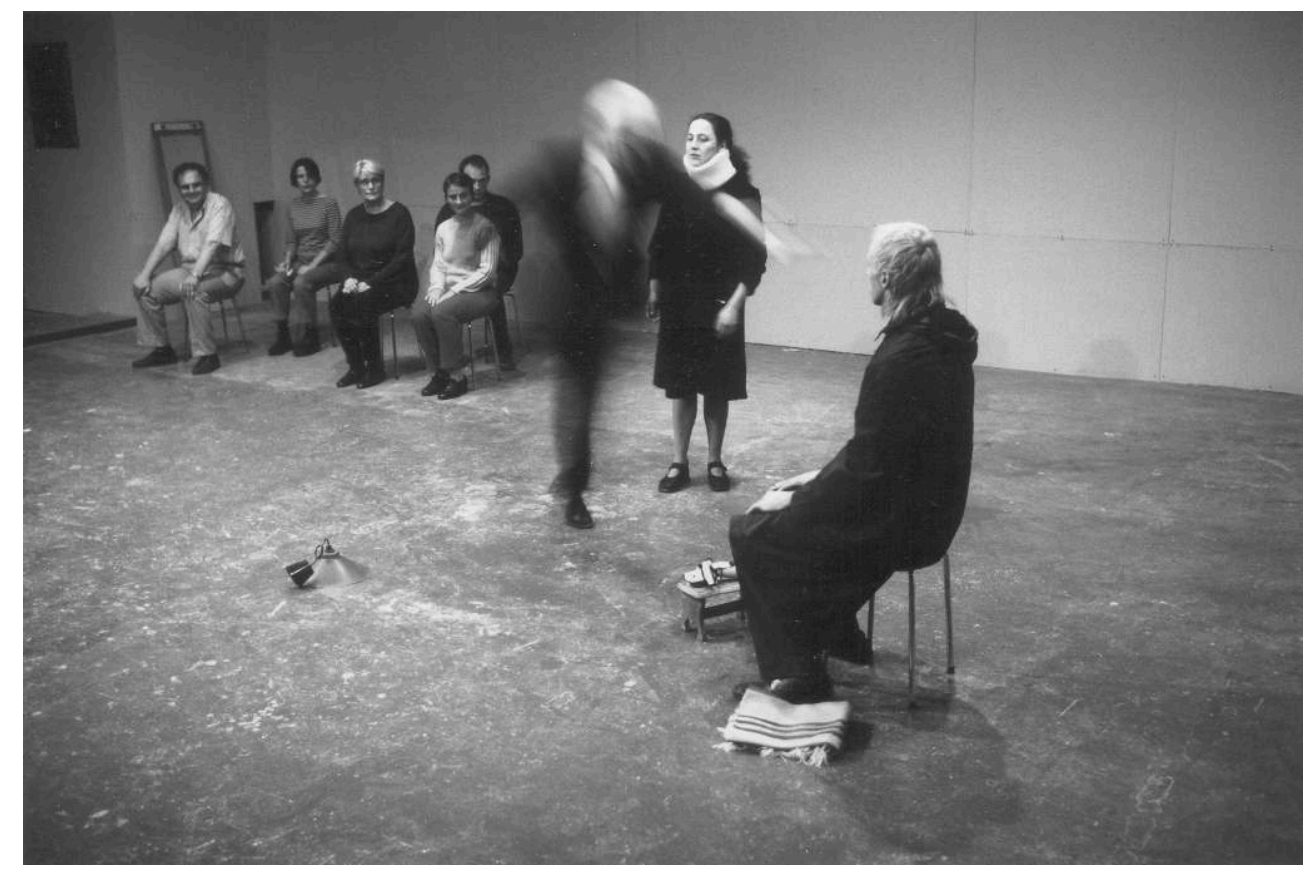

Florence Cuif

\section{La mémoire}

JACKY CASTANg : "Le rapport à la mémoire qui me semble être une parole déjà entendue, il y a des exemples: "Elle aura 100 ans en août", "Mon Dieu, la grande perche", est-ce que c'est quelque chose, qui est fortement ancré, imprimé en toi, ou est-ce que c'est quelque chose que tu as noté quelque part, à un moment donné. »

Philippe Minyana : «Ah ! Oui, oui, oui, j'ai toujours plein de calepins, je note plein de trucs que j'entends, ou des trucs que je lis. Je lis beaucoup de polars, et dans les polars il y a plein de phrases triviales comme ça. Je veux dire que oui, je pique plein plein de choses que je lis et je les écris sur des calepins et après je les réutilise. Pour revenir au thème de la mémoire, il y a une scène que j'aime particulièrement dans Pièces, c'est quand il revient dans son pays natal. On entend les poules tout ça, et tout le monde se retrouve, c'est les retrouvailles: "Ah! mon dieu le revenant, la grande perche". Et moi j'ai vécu cette scène quand ma mère est décédée. Je suis revenu l'année suivante dans le village, et j'ai remonté la rue, et tous les gens sont apparus : "Ah ! le Philippe, le Philippe", et donc j'ai travaillé là-dessus. Et je pense que je vais repartir de cette matrice : les retrouvailles, pour faire une autre pièce que m'a commandée Cantarella. Donc, il y a, comme ça, une série d'émotions empilées que je saisis très fort, que j'entends, d'odeurs, de choses que je note effectivement qui seront des matériaux à travailler. »

\section{Accessibilité et/ou fidélité à soi-même}

JACKY CASTANg : "Si on revient sur la notion de facilité ou d'accessibilité, on parle de cette scène-là, on parle aussi de la scène des deux cousines. Je crois que tout le monde apprécie 
ces scènes. C'est quelque chose qui peut intervenir sur ton écriture : te dire comment dans la pièce précédente cette scène-là a pu marcher? »

Philippe Minyana : " Non, non, ce n'est pas en ces termes, en tout cas pas : "marcher". Je ne dirai jamais ça, je me rends bien compte que des scènes sont plus ou moins abordables, plus ou moins drôles, plus ou moins clownesques. La scène des cousines dont tu parles, je l'ai travaillée. Mais je ne sais pas combien de versions j'ai fait de cette scène. Je voulais de toute façon qu'il y ait cette répétition des mots, puisque d'abord c'est une chose qui me frappe énormément dans la conversation courante ; que les gens aient 17 ans ou 80 ans, ils répètent quatre fois la même chose. Tout à l'heure, j'ai eu au téléphone des copains, metteurs en scène qui ont 31 ans; ils m'ont répété quatre fois la même chose. C'est le truc de l'émotion, la parole de l'émotion. Et ça dit et ça se répète. Écoutez bien autour de vous on ne dit jamais une info, on la dit quatre fois de suite. J'avais vu un spectacle de Langhoff à Nanterre, L'inspecteur général de Gogol. La liberté qu'avait prise Langhoff était magnifique. C'est une scène très brève dans la pièce, où il est question de robe à mettre. "Je veux mettre ma robe rouge" et "Tu mettras ta robe grise" dit la mère à la fille, et la fille dit "Ah! non maman, je ne mettrai pas ma robe grise et je vais mettre la robe verte". Et sur cette connerie-là, le metteur en scène l'a montée en boucle. Il a monté ça, il a monté la sauce, et dans la salle les gens hurlaient de rire. Donc c'est répété tout le temps, tout le temps, tout le temps, de plus en plus fortement. Donc je m'étais dit: "Ça je le mettrai quelque part, je ferai quelque chose". J'ai fait je ne sais pas combien de versions de cette scène jusqu'à ce que ce soit... C'est loin, souvent loin du bureau, que ça arrive, où on travaille. C'était dans un moyen de transport. Donc j'ai dit: "Ah !" mais j'ai presque poussé un cri, j'ai dit : "Ça y est je sais. Il va y avoir ces..., et puis au milieu il va y avoir le monologue de la bonne, qui est aussi une histoire vraie, que j'avais lue, et qui se suicidera après." Donc voilà, j'avais tout à coup ma structure. $C^{\prime}$ est pareil j'étais au restaurant chinois, je m'en souviens, rue Durantin à Paris $18^{\mathrm{e}}$. Et je l'ai vue ouvrir la fenêtre et se suicider. Donc il y a des choses comme ça qui arrivent, qui sont merveilleuses. J'ai plein de restaurants à Paris, des lieux où j'ai écrit telle phrase, où ça m'a donné telle idée, etc. Je sais qu'une phrase exacte dans Inventaires a été écrite à Ma Bourgogne, place des Vosges à Paris, comme si l'endroit avait catalysé quelque chose, son énergie propre et donné une impulsion au travail en cours. Mais je ne parle jamais au sens de l'efficacité, je suis toujours fidèle à moimême d'abord ; après, si le public aime ça tant mieux. »

\section{Réalisme et/ou minimalisme}

PUBLIC : «À propos de cette scène sur les cousines, c'est vrai que les reprises de phrases moi je ne les ai pas ressenties comme les reprises de phrases du début par exemple. Au début c'est une espèce de comique de répétition, c'est comme chez Molière, "Et que diable allait-il faire dans cette galère", et c'est vrai que l'histoire du meuble d'angle, tout le monde a ri, ça a été immédiat. Par contre, dans la scène que vous évoquez, dans les reprises, "La mère a 100 ans", "On est ligoté", j'ai trouvé ça vraiment très obsédant de la part de ces deux femmes, et ça révélait plein de choses, plein de frustrations ... »

Philippe Minyana : "Bien sûr c'est une scène terrible. »

PUBLIC : «Et moi, j'ai trouvé ça vraiment terrible. »

Philippe Minyana : « Mais c'est terrible, et oui. » 
PUBLIC : «Vraiment terrible. J'ai trouvé qu'il y avait la mort qui pesait à ce moment-là, sur cette scène, moi ça m'a bouleversé. Oui, à propos de ces phrases, il y a quelque chose qui m'a d'autant plus surprise, c'est qu'il y en a une qui dit, à un moment donné, une des deux cousines qui dit, "Pour une fois qu'on ne parle pas de la pluie et du beau temps", et alors justement on parle de la pluie et du beau temps, et... »

PHILIPPE MiNYANA : "Pas vraiment non, pas vraiment. Tac fait beaucoup d'aveux à ce moment-là sur son humiliation à être là. II dit aussi : "I'Europe construit des musées en veux-tu, en voilà », j'envoie mes petits messages comme ça, pouf, pouf, pouf. »

PUвьıс : «Et donc, moi je trouvais que les deux femmes parlaient parce que, pour elles, la parole, même si elle est banale, c'était aussi une manière d'exister. »

Philippe Minyana : «Bien sûr!"

PUBLIC : «Et moi, ça m'a rappelé une phrase de Lacan, quand il dit qu'il faut mettre le corps en mots pour éviter les maux du corps. »

Philippe Minyana : « Pas mal. »

PUBLIC : «Et là, vraiment, pour moi, cette phrase-là elle prenait du sens »

Philippe Minyana: "Oui, moi aussi je trouve ça terrible, hein, c'est horriblement grinçant. Mais les actrices sont tellement justes, comment dire tellement réelles. Il faut quand même que vous sachiez que la dame qui joue la cousine aînée, c'est pas une comédienne, c'est une amateur. Elle est tellement géniale qu'on lui a donné ce rôle ; elle est pilepoil, quoi, dans le rôle, on ne peut pas trouver mieux. Et quand je lui dis : "Tu es formidable Christiane, tout le monde te trouve formidable" ; "Oh ! mais c'est un rôle facile", elle me dit. "C'est un rôle payant", elle me dit, comme une vieille pro. »

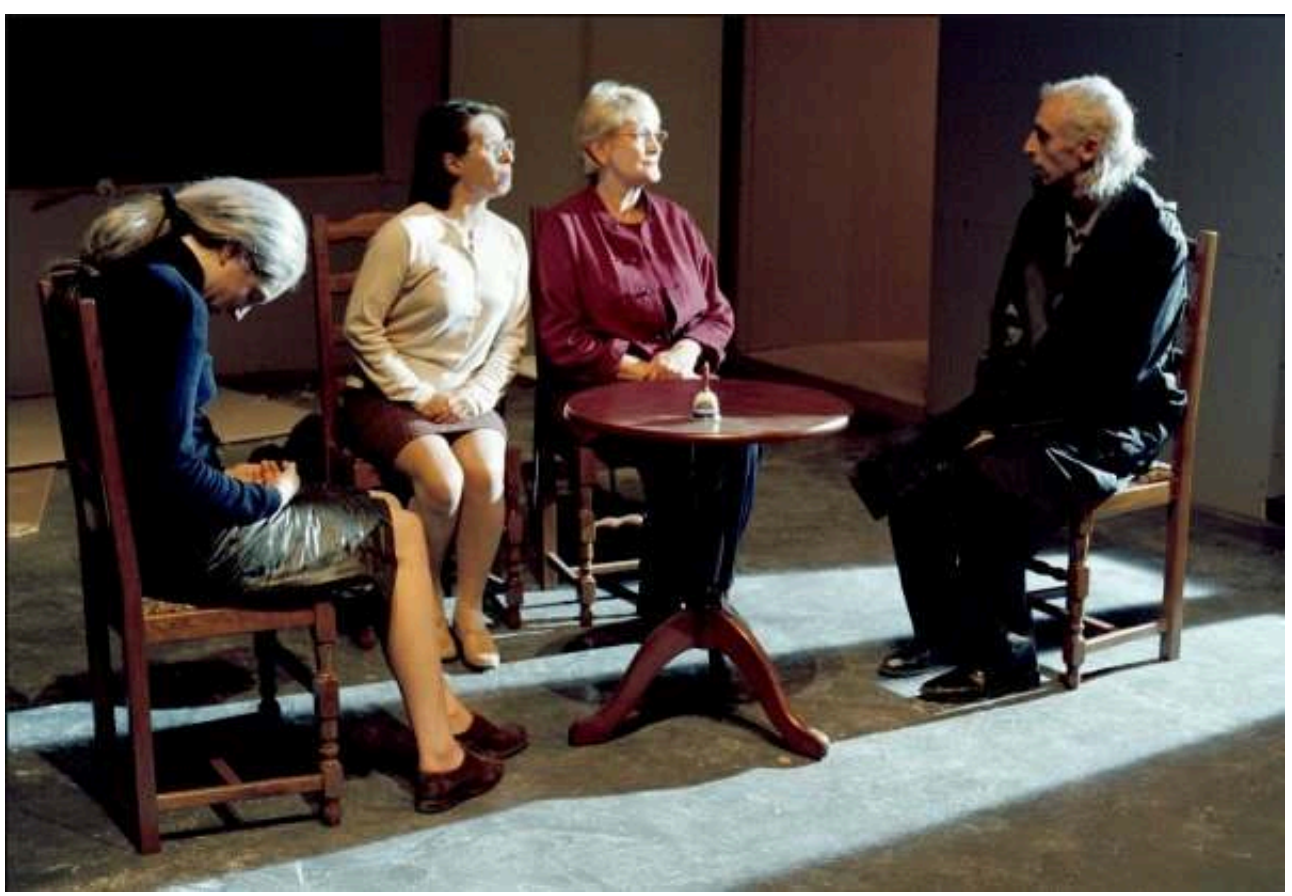

Cliché site web Minyana

PUBuIC : «Alors, pourquoi dans cette scène, le thé chaud »

Philippe Minyana : "Chocolat, je crois » 
PUBLIC : «le chocolat chaud fume dans les tasses, pourquoi avoir... »

Philippe Minyana : « Déjà qu'il n'y a pas de décor, hein, parce que, quand même, moi j'indiquais dans la didascalie: "un salon bourgeois avec le portrait du général de Gaulle". Les vieilles pouffiasses gaulliennes et donc, il n'y a pas de Gaulle, et il y a les débris de la scène d'avant, il y a quelques chaises simplement qui stipulent le salon bourgeois, donc on peut dire qu'il y a vraiment un minimalisme total et une stylisation totale. Au moins, qu'il y ait un élément de réel, elles boivent réellement le chocolat de cinq heures ; je pense que c'est une des raisons aussi. Comme il n'y a pas de reconstitution, la plupart des metteurs en scène français lambda auraient fait vraiment un salon bourgeois avec le portrait du général de Gaulle, comme il n'y a rien de tout ça, il faut quand même qu'il y ait des signes. Émettre des signes au théâtre quand même, pour qu'il y ait un minimum de réalité, de possible, que ça advienne, qu'on se dise ben oui c'est possible, c'est vraiment des cousines dans leur salon etc. Il suffit qu'il y ait une table, un guéridon... c'est ça qu'est merveilleux au théâtre, il suffit de trois éléments et on est dans un salon bourgeois. »

JACKY CASTANg : «La bonne?»

Philippe Minyana : « La bonne avec le costume, je veux dire, il y a des gravats autour. C'est ça le miracle au théâtre, on oublie les gravats. Et en même temps je trouve des bourgeoises qui boivent le thé dans des gravats, c'est pas mal aussi. Au niveau de ce que ça implique, et de ce que ça signifie sur le mortifère d'une société épuisée. »

PUBLIC : «Surtout qu'à ce moment-là un petit coup de chocolat chaud, c'était peut-être pas mal, quoi, la scène est tellement... Je trouvais que la réalité justement du chocolat qui fume était vraiment quelque chose à part, ce n'était pas... »

Philippe Minyana: "Oui, voilà, c'est un réconfort le chocolat chaud, c'est un réconfort..." "

PUBLIC : "Voilà on le ressent comme ça, parce qu'il y a une chape là qui tombe, un petit coup de chocolat chaud...»

Philippe Minyana: "Puis je trouve que c'est un rite de la visite: on sert à boire quelque chose aux invités. Donc je trouvais, c'est pourquoi je l'ai indiqué, qu'il y avait ce, cette chose qui se passait en tout cas, c'était signalé dans la pièce. Bon je ne sais pas si j'ai mis « chocolat ", certainement pas, mais en tout cas ils boivent le thé ou ils boivent quelque chose, quoi, ils boivent. Je trouvais que c'était important de, qu'on ait un reflet d'une réalité. ${ }^{10}$ 


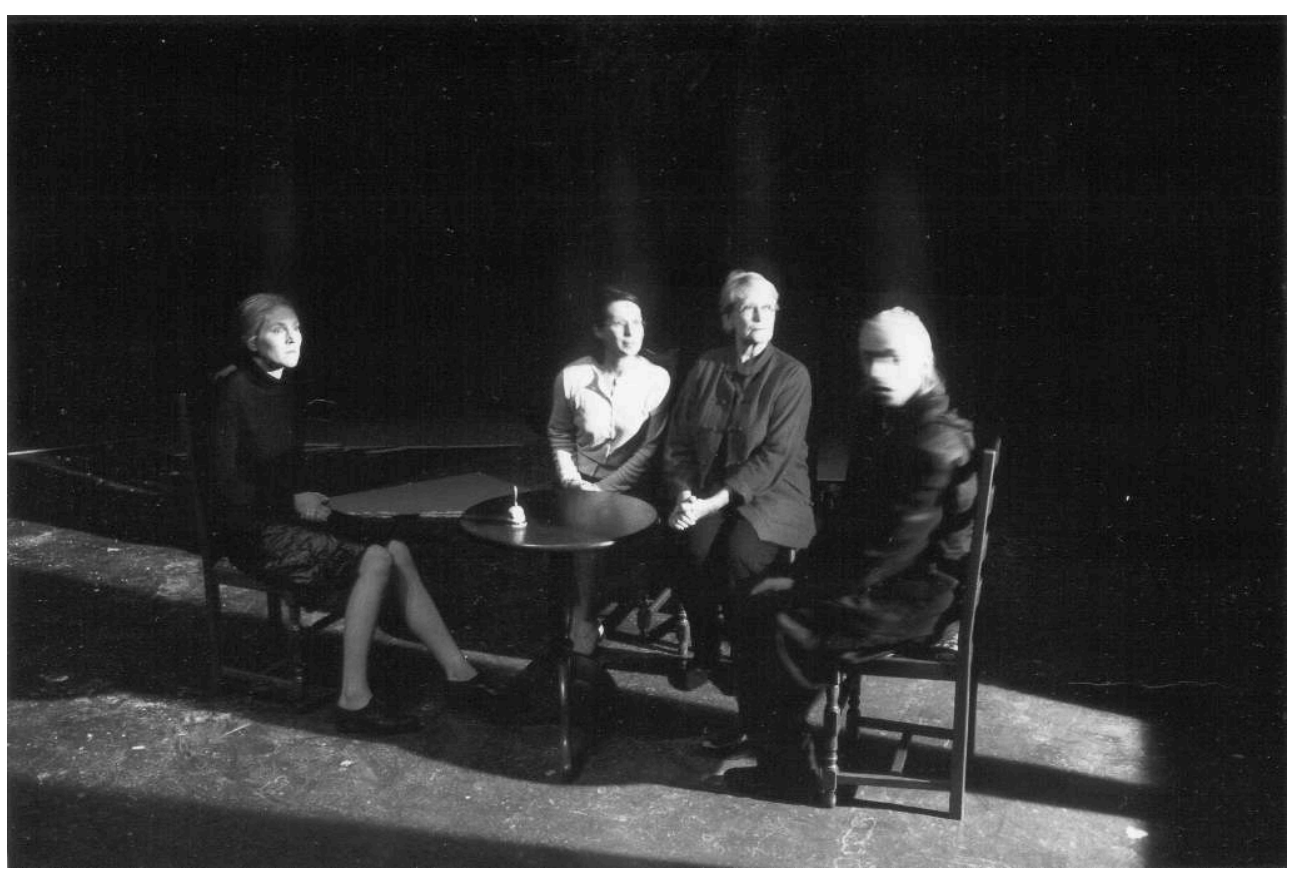

Florence Cuif

\section{Répétitions et composition musicale}

PUвLIC : «Au niveau de l'écriture justement sur ce genre de scène... Est-ce qu'il y a comme des fils pré-écrits par des personnages qui après s'entrecroisent: ou alors chaque phrase vient après l'autre?»

Philippe Minyana : «Oui, oui, l'une après l'autre. Ah, oui! Parce que, comment diraisje, ça joue ensemble, c'est de la musique. Il y a quelquefois trois mots ou un mot en trop ; quand on écrit on enlève beaucoup quand on écrit, on gomme, on recommence, beaucoup, beaucoup. Donc la phrase, comment dire, en soi, elle n'existe pas. Elle a besoin d'une autre phrase pour avoir son volume, sa densité, son énergie, sa force, sa direction. Il faut qu'il y ait donc toute la page, que je la visualise, que je la dise à haute voix pour voir comment ça fait rond, comment ça se structure, comment ça s'architecture, comme une musique, comme un dessin d'architecture. Comment si je mets la poutre, j'ai besoin de la tuile ou j'ai besoin, s'il y a un muret, j'ai besoin de l'escalier, vous voyez ce que je veux dire, il y a une espèce d'harmonie secrète qui doit être révélée. Et mon grand souci quand j'écris c'est ça : il faut que ça chante, que ça bouge, que ça bruisse, sinon c'est de la télé, c'est du dialogue, qui tombe comme une pierre dans la marre. Il n'y a pas de grâce, il n'y a pas de mouvement, il n'y a pas de musique, il faut que ça avance, il faut que ça avance. Donc toutes les répliques fonctionnent ensemble, donc obligatoirement, j'écris des ensembles. »

PUBuIC: "Je voulais revenir sur ce que vous faites dire à Tac au début de la pièce, que quand on dit les mots on peut avoir des nuances. Ça m'a fait penser à Nathalie Sarraute et à son travail sur le langage. Et je voudrais savoir le rapport entre toutes ces répétitions, ces redites, ces radotages aussi, est-ce que c'est une manière d'essayer de retrouver la nuance des mots ou... »

PhilipPe minyana : "C'est plutôt pour rendre compte d'un état de la parole, de la prise de parole des humains, c'est-à-dire la parole de l'émotion. Or la parole de l'émotion, elle est. Ce n'est pas la parole du speaker, de l'homme politique, du médecin qui sont carrés, rationnels, 
calibrés, formatés. C'est de la parole en vrac, en désordre, c'est de la parole qui reprend, qui se redit, qui se redit encore, et là, je l'ai poussée au maximum évidemment. Mais, je pense que ce n'est pas loin d'une sorte de réalité. Vous savez quand on cadre le réel très fort, qu'on le met sous la loupe, ça paraît invraisemblable, or c'est du réel. Si on met une caméra de surveillance, ou un magnétophone dans un repas de famille, ou une réunion d'amis, on l'a peut-être déjà fait, vous l'avez peut-être déjà fait, c'est très surprenant. Les gens radotent tous. Radoter c'est pas le mot, c'est-à-dire on reprend tout le temps ce qu'on a déjà dit et on monte en boucle trente phrases. J'ai un ami qui m'appelle, il vient de créer un spectacle, il est ému, et il me dit la phrase trois fois de suite : "Mais faut dire, tu sais, j'ai eu beaucoup de problèmes, tu sais parce que j'ai eu vraiment beaucoup de problèmes, parce que ce spectacle, quand même, ça m'a créé beaucoup de problèmes". Quand il est plus calme et qu'il répète encore trois fois, je lui dis : "Tu me l'as déjà dit trois fois", il me dit "Oui, oui, oui, oh ! Excuse-moi mais, ah ! Ça me stresse ce truc, voilà." Je crois que c'est rendre compte de l'émotion des humains. C'est ça qui m'intéresse au théâtre, c'est de donner, d'envoyer des images comme ça, ou des situations qui vont montrer l'humain dans sa fragilité. Dans la scène des cousines, elle est très travaillée musicalement. II y a des redites mais il y $a$, chaque fois, à chaque mouvement, il y a une info en plus qui arrive. II y a tout le temps une autre note qui arrive et qui constitue le portrait de tous ces gens qui sont là. La sœur qui dit des choses nouvelles chaque fois, elle fait avancer la scène; Tac dit aussi sur son humiliation, sur son manteau qui pue, sur les musées, sur quand on est sur un quai on entend des petites phrases; tous les deux font avancer la scène, pas les cousines, quoique... elles font des aveux, petit à petit aussi, elles se dévoilent. »

\section{La fuite et l'errance}

PUвьıс : "Je pense que les gens, j'en ai parlé avec mes amis, les gens l'ont ressenti vraiment comme quelque chose de douloureux. »

Philippe Minyana : « Mais c'est douloureux, c'est très douloureux. »

PUBLIC : « Mais quand vous dites les gens répètent trois fois, mais moi je le constate autour de moi, dans mes associations, dans toutes mes activités, mais il faut simplement se poser la question, parce que l'autre en face n'écoute pas. »

Philippe Minyana : « N'entend pas, n'écoute pas, oui, oui, c'est vrai. »

PUвLIC : "N'entend pas et ne veut pas entendre. »

Philippe Minyana : « Oui, oui, oui.»

PUBlı : « Vous avez très peu de gens qui savent écouter, faut pas s'étonner. »

Philippe Minyana : "C'est vrai, c'est vrai, pis comme on est habitué au zapping. Moimême j'étais à l'hôtel à Munich, je n'ai pas pu regarder un seul truc vraiment. J'ai passé, j'ai zappé et c'était génial, je prenais un pied pas possible, j'étais là et j'ai zappé tous les soirs comme ça. Pendant deux heures je zappais, il n'y a rien qui me retenait. »

PUвLıc : "Oui, mais ce que vous dites là pour l'œil, eh bien dans la réalité, dans la société les gens se sentent. »

Philippe Minyana : « Absolument oui, oui, c'est vrai, des autistes. »

PUвьıс : «Faut pas s'étonner. Je suis désolée. »

Philippe Minyana: «Mais non! Écoutez. Non. C'est formidable que vous parliez, madame.»

PUвLıc : "À un moment donné, quand à la fin, ils disent que la fille de Tac, elle est morte, elle a été défenestrée, est-ce que c'est la bonne, sa fille?»

Philippe Minyana : «Ah non, non, non, non. » 
PUBLIC : «Est-ce que ça a un rapport? »

Philippe Minyana : «Elles se défenestrent toutes, Odile s'est défenestrée, sa fille s'est défenestrée, est tombée, non, elle s'est pas défenestrée, elle est tombée et la bonne se défenestre effectivement. $»^{11}$

PUBLIC : « Mais ça a un rapport ou non?»

Philippe Minyana : «Non! Non! C'est le hasard que tout le monde se défenestre. C'est une façon d'en finir. Non! non! il n'y a pas de rapport. Oui, évidemment, on a un système d'empilement. J'ai remarqué que, depuis quelque temps, dans les journaux, il y a trois façons de se suicider : il y a les gens qui se pendent, mais ça c'est à la campagne plutôt, et, à la ville, on se défenestre parce qu'il y a des immeubles assez hauts. Et puis, il y a cette mort que, d'ailleurs, suggère Tac en se mettant le plastique sur la tête, parce que le peintre Buffet, Bernard Buffet est mort comme ça. Il s'est pris des somnifères, il s'est mis un plastique autour de la tête, il s'est mis un truc, et il est mort étouffé, y a pas longtemps l'année dernière. Donc ça m'avait frappé, et voilà c'est tout c'est simplement un mode d'emploi, je dirais, de nos sociétés. Comment on aime ou comment on n'aime pas, comment on relationne, comment on demande, comment on ne supporte plus de vivre, etc. et quelles sont les conséquences de tout ça. »

PUвbIC : "Ce qui fait que sur la défenestration, il y a une façon qui est tout à fait logique dans ce thème de l'expropriation et que... »

Philippe Minyana : En plus oui, mais ça je n'y avais pas pensé, voyez, vous me révélez quelque chose là, parce que je n'y avais pas du tout pensé. C'est-à-dire que tout tourne autour des corps sans logis. Toute la pièce parle de la perte, ceux qui ont perdu leurs enfants, celui qui a perdu ses yeux d'ailleurs, tout le monde a perdu son logement, sa maison, sa région, sa famille. Ce thème de l'errance est un thème qui moi me bouleverse particulièrement. C'est vrai que quand on ouvre le journal, la télé, on ne voit que des errants : en ce moment, les Afghans évidemment bien sûr, mais pas qu'eux. Il y a toujours eu des errants, avant il y avait les Kosovars, avant il y avait les Croates, avant il y avait les Grecs, avant il y avait les Argentins, il y a toujours eu des peuples en errance. C'est biblique, ça, et je pense que c'est une de nos légendes, l'errance, les peuples qui errent, qui ont franchi les portes du confort organisé. Et c'est une chose, une légende que je trouve très vive, toujours bonne à revisiter. Ça, j'en ai conscience, quand je l'écris, c'est de ça dont je veux parler. »

PUBLIC : «À ce propos un article de Charlie Hebdo, de ces dernières semaines, évoquait le nombre d'appartements disponibles en île-de-France qui était de l'ordre de 400 000, et la capacité extraordinaire des politiques à découvrir en fin d'année la détresse et la difficulté à en héberger quelques milliers, mais bon il y a certainement un rapport avec ce mouvement migratoire de toujours... »

Philippe Minyana : « Absolument. »

PUвцIC : «...qui explique que les politiques ont sûrement intériorisé le mouvement perpétuel et pour ne pas l'interrompre, l'observent et continuent à l'observer. Toujours est-il que Robert Cantarella évoquait le rapport à des tableaux moyenâgeux dans la scène où la cloison... »

Philippe Minyana : « La plaine. »

PUвиı : «...est abattue, il faut quand même... j'ai entendu l'évocation quoi, autrement ça me serait passé au-dessus de la casquette. »

Philippe Minyana : « Ben d'ailleurs tant mieux. » 


\section{Douleur et/ou apaisement}

PUвLIC : " Je n'ai pas tout compris... »

Philippe Minyana : «Tant mieux parce que c'est pas suffisamment volontaire, c'est un clin d'œil seulement... »

PUBbıc : «Y a plein de clés, quoi, y a plein de clés, mais l'essentiel n'est pas tant de les avoir...»

Philippe Minyana : «C'est de recevoir ce qu'on donne ... »

PUBцic : «...c'est d'aller voir ce qu'il y a derrière la porte, ou la fenêtre, mais plus de sortir perplexe, de s'interroger, de rechercher...»

Philippe Minyana : «Oui, je crois ou de lire le texte, de venir le revoir à Besançon. À Paris il y a des gens qui l'ont vu trois fois le spectacle. Des jeunes étudiants de théâtre à Censier, ils sont venus trois fois pour bien saisir tout, pour bien voir tout, parce qu'ils avaient aimé. Ils avaient été touchés, donc ils sont revenus et je crois que, et quand on le revoit, on n'a pas la même sensation. D'ailleurs, ils me disaient toujours : "Ben c'est pas le même spectacle, c'est curieux." C'était le même, exactement le même, alors c'est vrai qu'entre chaque soir ça varie un peu, mais très peu. Et ils n'avaient pas l'impression de voir le même, c'est bizarre. Parce qu'ils n'avaient pas entendu certaines choses qu'ils ont entendu là, ils en ont entendu d'autres, ils ont fait leur choix et c'était jamais le même choix. Mais peu importe. Je veux dire, moi, que les trucs médiévaux, ça c'est le secret de la cuisine du scénographe, on s'en fout. Simplement, c'est vrai que c'est un clin d'œil. On peut remarquer ou pas le côté sujet religieux presque, où on a les figures comme ça, les errants tous réunis, peu importe, hein. Mais c'est beau ce que vous avez dit sur les migrations, les flots migratoires. »

PUвLIC : «Ça m'a fait prendre conscience que, ma femme me disait que j'étais un peu comme ça que je radotais beaucoup et puis, ça m'a, je réfléchissais à ça ces derniers jours, et puis je me rassure en me disant que c'est le cas de plein de gens qui nous entourent... »

Philippe Minyana : «C'est la norme. Moi aussi je radote»

PUBLIC : «Le film à la fin, c'est quelque chose dont vous avez discuté ?»

Philippe Minyana : «Non! non! non! Ça ils ont discuté entre eux avec le scénographe et le metteur en scène. Moi j'ai appris que ça allait se faire comme ça, mais je... Non! ce n'est pas du tout dans le texte ça, ni d'ailleurs le narrateur qui raconte toute la scène, et que ça se joue en décalé c'est pas dans le texte non plus. Ç'est les inventions de la mise en scène. Quant au film, ça c'est beau, le film... parce que c'est quinze ans après, et c'est la fin de cette histoire, et c'est une histoire de tous les temps. C'est un homme qui perd pied, qui ne sait plus où il est, qui cherche son fils. Je trouve que c'est assez beau de l'immobiliser comme une archive, comme une chose comme ça, qu'on pourra repasser à l'infini. Cette scène-là, moi je trouve que c'est très intelligent, en tout cas, ça perturbe le spectateur, mais c'est très intelligent. Je trouve ça assez beau ce travail, en plus il met Mahler dessus, ce qui est quand même pour Cantarella très nouveau. »

PUBLIC : « Moi j'ai trouvé ça très apaisant, parce qu'après toute la violence et ce que j'ai aimé aussi, c'est les comédiens qui regardent l'image... »

Philippe Minyana : « Et qui se doublent. » 
PUBuıc : «...et qui se doublent et ça faisait comme un accompagnement de mourant. Et l'avant-veille j'avais vu le film C'est la vie. Et c'est marrant, parce que j'ai trouvé le même accompagnement de celui qui perd les pédales, qui va quitter le monde. Mais c'était un apaisement, en même temps douloureux bien sûr, j'ai trouvé ça vraiment très beau... »

Philippe Minyana: «Mais c'est doux, oui, c'est vrai que vous avez raison, c'est doux, oui. C'est nouveau aussi chez Cantarella, et c'est pour ça aussi que je suis ému, parce que c'est des nouvelles armes, de nouvelles grammaires dont il se sert. Et aussi avec l'âge, bon il a 44 ans, il n'est pas très vieux, mais je trouve qu'il s'adoucit lui. Il y a moins le côté "provoc", le côté formel, le côté pied de nez qu'il avait souvent l'habitude de faire, et qui dérangeait le spectateur. Là je trouve qu'il y a une espèce d'harmonie, finalement dans tout ce spectacle, un petit peu comme un livre d'images, même s'il y a des difficultés de compréhension quelquefois. J'ai l'impression qu'on ouvre un album, un almanach Vermot et qu'on raconte l'histoire de ce vieil homme qui traverse les territoires pour trouver sa famille, sa sœur, ses cousines. Qui va dans un endroit pour chercher un petit studio à louer, puis, pan il y a une exposition des gens dans les goulags, le vieux en chaise roulante, la femme trop bien habillée, l'homme habillé en femme, tout à coup il arrive dans l'enfer. J'aime bien ces idées de grandes mythologies, le purgatoire, l'enfer, le paradis. Je trouve que ce sont de belles mythologies à traverser, à revisiter autrement et pour moi, Tac entre dans l'enfer. Chaque fois c'est un petit enfer. "

PUBuIC : "Oui puisqu'en fait il avait pas besoin de repères il avait les siens dans cet appartement où il avait...»

Philippe Minyana: "Bien sûr. Mais une chose, qui s'est passé à Paris qui est extraordinaire. C'est un soir qu'il y avait beaucoup de monde et des gens assez émus d'ailleurs, il faut le dire. Et puis j'étais dans le hall avec des amis du métier et une journaliste, et puis il y a une dame qui arrive vers moi en sanglots, qui me dit: "Oh! Monsieur Monsieur si vous saviez comme je suis bouleversée". Je dis : "Ah ! bon! Écoutez c'est très bien". Mais elle pleurait, mais vraiment elle sanglotait, et elle me dit "j'étais la voisine de ce monsieur. C'est moi qui l'ai aidé à se consoler, qui l'ai soigné, qui ai demandé aux commerçants de rendre les meubles. C'est moi qui étais son amie", elle me dit. En fait, il s'était réfugié chez une parente de sa mère, comme il n'avait plus d'appart, plus d'affaires plus rien. Et alors, elle était comédienne cette dame, et c'est une amie à elle qui lui a dit: "Mais tu sais l'histoire que tu m'as racontée, il y a dix ans, de ce vieil homme, ça m'avait frappé, Minyana en a fait une pièce." Donc, elle est venue voir la pièce. »

\section{NOTES}

1. L'enregistrement et la transcription ont été assurés par le théâtre Edwige Feuillère. La ponctuation, par la rédaction de Coulisses.

2. Drames Brefs (1), Éditions Théâtrales, 1995.

Drames Brefs (2), Éditions Théâtrales, 1997.

3. Chambres, Théâtrales / Edilig, 1988, rééditions aux Éditions Théâtrales, 1993. 
4. Inventaires, Avant Scène, $n^{\circ} 809,1987$, reprise avec des modifications aux Éditions Théâtrales, 1993.

5. Les Guerriers, Théâtrales / Edilij, 1988, rééditions aux Éditions Théâtrales, 1993.

6. La Maison des morts, Éditions Théâtrales, 1996.

7. Pièces, Éditions Théâtrales, mars 2001.

8. Sur le travail de Philippe Minyana et de Robert Cantarella, voir Philippe Minyana ou La Parole visible.

9. La «maison des nains" n'est pas présente à chaque représentation, en fonction de la dimension du plateau. Elle n'y était pas à Besançon.

10. N. D. L. R. Pièces, Éditions Théâtrales, p. 115.

Solange dit "Voulez-vous boire quelque chose?" Elle sonne. La bonne entre avec un plateau de boissons, pose le plateau et sort. Solange fait le service. Ils boivent.

11. N.D.L.R. Pièces, op. cit., tableau 8, Habitants parlents (2), p. 123, « il retrouve sa fille elle picole un peu et elle tombe du cinquième C'est pas vrai si si c'est comme ça... Et sa fille tombée du cinquième. » 